\title{
UBVRIJHK Photometry of Short-Period Red Variables Identified by the Hipparcos Survey
}

\author{
C. D. Laney, C. Koen \\ South African Astronomical Observatory, PO Box 9, Observatory 7935, \\ Cape, South Africa
}

\begin{abstract}
The Hipparcos survey team discovered thousands of new variables, including $M$ giants with periods as short as a day, which appear to have maintained rough phase coherence over a period of more than three years. New optical and infrared photometry has been obtained as part of an effort to understand the nature of these anomalous stars.
\end{abstract}

New $U B V R I J H K$ photometry was obtained between December 1998 and February 1999 for 18 periodic red variables from the Hipparcos catalogue with Hipparcos periods less than 12 days. Two are chromospherically active $\mathrm{K}$ dwarfs, two are early $\mathrm{K}$ giants, and the remainder are $\mathrm{K} 5$ to early- $\mathrm{M}$ giants. The average number of observations is $18(J H K)$ and $20\left(U B V R I_{C}\right)$. The $J H K$ average just cited excludes two stars observed only 3 times each. A few (9-10) $J H K$ observations have also been obtained for two short-period M-giant variables identified by Woitas (1997).

The $B V I_{C}$ colors of the 14 late-type giants are typical for $\mathrm{K} 5$ to early $\mathrm{M}$ giants, and do not indicate significant reddening. The $J H K$ colors are similar to those of the bluest mass-losing stars in the South Galactic Cap (Whitelock et al. 1995), and to those of the longer-period semiregular variables in metalrich globular clusters (Whitelock 1986). The $H-K$ colors in our sample of 14 late-type variables are bluer than for short-period Miras and Mira-like variables (Whitelock et al. 1999), and most of these 14 stars occupy a region in the $H-K, J-H$ two-color diagram which is a redward extension of the sequence defined by nonvariable giants in the SAAO list of infrared standard stars. The $\mathrm{K}$ giants and dwarfs all have redder $H-K$ colors than typical $\mathrm{K}$ giants at the same unreddened $J-H$, forming a sequence parallel to the locus of most $\mathrm{K}$ stars. The IRAS colors, as with most short-period red giant variables (Koen \& Laney 1999, submitted, hereafter KL), show no evidence of significant dust.

In an independent analysis of the Hipparcos photometry for 'periodic' shortperiod M-giant variables, KL found that the principal period present was the same as found by the Hipparcos team in most cases, but that there appeared to be other periods present as well. When the periods identified by KL (or by the same methods, for stars not considered by KL) are applied to the 1998/99 optical photometry, $\sigma_{V}$ is reduced for 16 of the 18 stars observed. While the amplitudes in the new data are not necessarily identical, the agreement for most stars is as good as one could reasonably expect for limited data and periodic but semiregular variables. Four stars show signs of variability on time scales longer than the periods listed by KL. One shows periodic behavior at the KL periods superimposed on a slow drift; one shows marginal short-period variability com- 


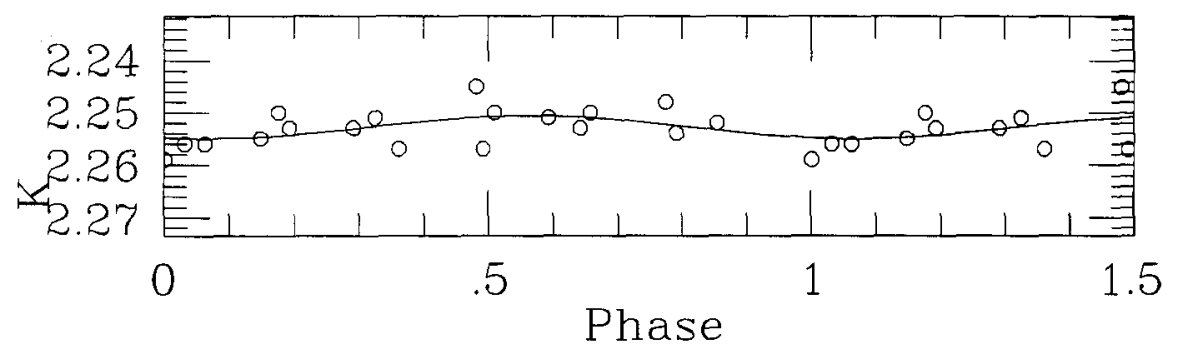

Figure 1. The $K$ light curve for HIP 52789, folded at the Hipparcos period of 6.693 days.

bined with a period of about 19 days; one shows only a slow drift in brightness; one star shows apparent evidence of period doubling at the longest KL period.

In the near-infrared, however, evidence of short-period M-giant variability is marginal at best, despite typical $\sigma_{K}$ values of 0.006 or less in the observations (Fig. 1). For only 4 of the $12 \mathrm{~K} 5$ to early-M giants with reasonable data was $\sigma_{K}$ (assuming the KL periods) reduced by more than 0.001 compared with assuming no short-period variability. Even for the four marginal cases where the scatter was slightly reduced, the $K$ semi-amplitudes were larger than 0.01 only for two stars where most of the variation appeared to be at periods (found by KL but not by Hipparcos) of 36 and 88 days. A comparison of the $V$ vs. $K$ amplitudes shows that the $K$ semi-amplitudes are very low regardless of the $V$ semi-amplitude except for periods over 20 days - and except for the four early- $K$ dwarfs and giants. The two known chromospherically active dwarfs have amplitude ratios near those for Mira-like Hipparcos variables (Whitelock, Marang, \& Feast 1999) and for Cepheids. The two $\mathrm{K}$ giant variables show amplitude ratios nearer to unity. They may be ellipsoidal variables. Of these, HIP 24318 is listed as a binary/multiple star by SIMBAD; HIP 45633 may be similar. KL show that the short-period $\mathrm{M}$ giant variables are probably pulsating in high overtones, with a strong correlation between $\mathrm{Q}$ and period. Very low $K / V$ amplitude ratios are certainly consistent with what is seen in hotter stars pulsating in high overtones.

Observations of two short-period M-giant variables from Woitas (1997) also showed no obvious variations at $K$. In a reanalysis of the Tycho data, we were unable to recover the short periods cited by Woitas, and for HD 95687 the only variations clearly present were at 'periods' so long that it was not possible to establish from three years of data whether the variability was periodic or random.

\section{References}

Whitelock, P. A. 1986, MNRAS, 219, 525

Whitelock, P. A., Marang F., \& Feast M. W. 1999, preprint

Whitelock, P. A., Menzies J. W., Feast M. W., Catchpole R. M., Marang F., \& Carter B. S. 1995, MNRAS, 276, 219

Woitas, J. 1997, IBVS, No. 4444 